\title{
Caracterización anatómica de la madera y carbón de cinco especies leñosas para su identificación
}

\author{
Anatomical characterization of wood and charcoal of five \\ wood species for their identification
}

\author{
Karina Bustamante ${ }^{1, *}$, Manuel Chavesta ${ }^{2}$ y Héctor Gonzales ${ }^{2}$
}

\begin{abstract}
Recibido: 02 noviembre 2018 | Aceptado: 24 junio 2019 | Publicado en línea: 15 diciembre 2019 Citación: Bustamante, K; Chavesta, M; Gonzales, H. 2019. Caracterización anatómica de la madera y carbón de cinco especies leñosas para su indentificación. Revista Forestal del Perú 34(2): 144-162. DOI: http://dx.doi.org/10.21704/rfp.v34i2.1326
\end{abstract}

\begin{abstract}
Resumen
El estudio tuvo como finalidad describir y caracterizar la estructura anatómica de la madera y el carbón de las especies capirona (Calycophyllum spruceanum (Benth.) Hook. ex Schum.), copaiba (Copaifera officinalis L.), shihuahuaco (Dipteryx micrantha Harms.), mango (Mangifera indica L.) y naranjo (Citrus sinensis (L.) Osbeck) y comparar las características antes y después del proceso de carbonización. Dichas especies fueron seleccionadas para la investigación, debido a que después del carbón de algarrobo son los carbones de las especies más comercializados en el mercado peruano, incluidos los frutales mango y naranjo. Las muestras de madera se carbonizaron en una mufla a $450^{\circ} \mathrm{C}$ por una hora. Después de la carbonización se determinó que los elementos leñosos como el parénquima axial, canales gomíferos y anillos de crecimiento se conservan y permiten la identificación de las especies tal y como ocurrió para las especies estudiadas. Si bien en copaiba no se pudo observar el parénquima vasicéntrico, características anatómicas como los canales gomíferos permitieron su identificación; asimismo, existe una reducción del tamaño de los poros y un incremento en el número de poros $/ \mathrm{mm}^{2}$ en las cinco especies estudiadas. Las gomas no se conservan después de la combustión; sin embargo, la tilosis sí lo hace. El análisis físico-químico al carbón determinó que la madera de mayor densidad no siempre produce carbón más denso a consecuencia de la presencia de componentes volátiles. Se evaluaron muestras de carbón molido de capirona y shihuahuaco en un microscopio electrónico de barrido, donde a pesar del aumento del equipo no se llegó a visualizar el parénquima difuso de la capirona; no obstante, se pudieron observar otras características, lo que permitió diferenciarlas de otros tipos de carbones.
\end{abstract}

Palabras clave: carbón vegetal, carbonización, anatomía de la madera, identificación de especies, microscopía electrónica de barrido

\footnotetext{
${ }^{1}$ Consultor Independiente.

${ }^{2}$ Departamento de Industrias Forestales, Facultad de Ciencias Forestales, Universidad Nacional Agraria La Molina (UNALM), Av. La Molina s/n, La Molina, Lima, Perú.

* Autor de Correspondencia: karina.bdr@gmail.com
} 


\begin{abstract}
The purpose of this study was to describe and characterize the anatomical structure of the wood and charcoal of capirona (Calycophyllum spruceanum (Benth.) Hook. ex Schum.), copaiba (Copaifera officinalis L.), shihuahuaco (Dipteryx micrantha Harms.), mango (Mangifera indica L.) and orange (Citrus sinensis (L.) Osbeck) trees, and to compare the characteristics before and after the carbonization process. These species were selected for this study because their charcoal are the most commercialized in the Peruvian market after the algarrobo's charcoal, including the fruit species mango and orange. The wood samples were charred in a muffle at $450^{\circ} \mathrm{C}$ for one hour. After carbonization it was determined that the woody elements as axial parenchyma, gum canals and growth rings, are preserved and allow the identification of the species like it happened for the studied species. While the vasicentric parenchyma wasn't cospicuos in the case of the cabonized copaiba, other anatomic features like its gum canals made its identification possible; likewise, there is a reduction in pore size and an increase in the number of pores $/ \mathrm{mm}^{2}$ in the five analyzed species. The gums are not preserved after carbonization, however the thyloses does remain. The physical-chemical analysis to the charcoal determined that the wood with the greatest density does not always produce charcoal with great density as result of the presence of volatile components. Samples of capirona and shihuahuaco ground charcoal were evaluated in a scanning electron microscope, whose zoom couldn't help identify the capirona's diffuse parenchyma; nevertheless, other features were noticed, which allowed to differentiate them from other types of coals.
\end{abstract}

Key words: vegetable charcoal, carbonization, wood anatomy, species identification, scanning electron microscopy

\section{Introducción}

En la última década los bosques secos del norte han sido los principales productores de carbón vegetal en el Perú, abasteciendo mayormente con carbón de algarrobo (Prosopis sp.) especie en veda desde 1974, cuya extracción mayormente se realiza de manera ilegal; sin embargo, a partir del año 2012 la situación cambió, ya que Ucayali incrementó su producción de carbón a consecuencia del mayor aprovechamiento de los residuos generados en los aserraderos, principalmente de especies duras como shihuahuaco, capirona y copaiba (DGFFS 2014). Encuestas realizadas a carboneros de Pucallpa, confirman la preferencia de las pollerías (principales consumidores) por adquirir carbón de especies como algarrobo y shihuahuaco resaltando entre sus características el mayor tiempo de combustión, bajos niveles de cenizas, aroma y sabor particular a los alimentos (Bennett-Curry et al. 2013). A consecuencia de la preferencia del consumidor por estas especies, los carboneros realizan denodados esfuerzos para poder cubrir la demanda, sin importar que el aprovechamiento se realice de forma ilegal, incluso comercializándose otras especies con el nombre de algarrobo, siendo perjudicado el consumidor final, ya que no recibe el producto con las características deseadas.

Ante el comercio desleal, la explotación excesiva de algunas especies preferidas por el consumidor y el ingreso al mercado de especies frutales como el carbón de mango y naranjo, se vuelve necesario determinar la identificación del carbón para diferenciar las especies de aquellas que se encuentran en estado vulnerable como el algarrobo, lo que será de gran ayuda a la autoridad forestal encargada de supervisar e identificar los cargamentos de carbón que se transportan. Siendo de interés contar con patrones que permitan la identificación del tipo de madera empleada, creando una colección que sirva para auxiliar en la identificación del carbón.

El primer estudio de identificación de carbón vegetal en Perú fue realizado por Pipa et al. (2009), quién validó una metodología de identificación del carbón vegetal del género Prosopis (algarrobo) a partir de su estructura 
anatómica. En dicho estudio se carbonizaron muestras del género Prosopis y otras especies testigos como aguano masha (Machaerium inundatum (Mart. ex Benth.) Ducke), quinilla (Manilkara bidentata (A. DC.) A. Chev.), eucalipto (Eucalyptus globulus Labill.) y olivo (Olea europaea L.). Dicho trabajo demostró que el tipo y distribución de poros, así como el tipo de parénquima axial son características que perduran después del proceso de carbonización y permiten la identificación del carbón; mientras que la presencia de inclusiones como gomas y tilosis son características que no pueden ser utilizadas, ya que se pierden post-carbonización.

En ocho especies comercializadas como carbón en la Amazonia brasileña se reporta que el parénquima y los elementos vasculares de algunas especies fueron difíciles de observar con lupa de 10x, como consecuencia de la contracción volumétrica. En algunos casos los canales secretores axiales, la obstrucción de vasos y los anillos de crecimiento se mantuvieron después de la carbonización, permitiendo la identificación a nivel de especie. La presencia de grietas se manifestó en la orientación de los radios en la mayoría de especies y algunas veces fueron en la dirección del parénquima axial, ello se atribuye a la baja densidad de la especie; asimismo, la descomposición rápida de la superficie de la madera por acción del calor y por la presión que causa la liberación de gases por pirolisis fueron otras de las razones de la presencia de grietas. Dichos gases combinados con la fragilidad de las estructuras celulares, especialmente del tejido parenquimático, ocasionan el colapso en la madera; no obstante, se afirma la posibilidad de identificar el carbón a nivel de familia y género con lupa de 10x y de un estereomicroscopio de luz reflejada con aumentos de 10 a 25x (Souza et al. 2015).

Gonçalves et al. (2015), a través del estudio macroscópico realizado a 25 maderas brasileñas, mencionan que las principales características anatómicas que se utilizan para identificar el género del carbón vegetal se encuentran en la sección transversal, se mencionan al parénquima axial, el diámetro y distribución de vasos y ancho de radios; no obstante, mencionan que la característica más importante para la identificación de la madera es el parénquima axial, aunque en carbón no siempre es fácil visualizarlo, especialmente cuando el parénquima es escaso y/o difuso; en tanto, que el parénquima en bandas y los diferentes tipos de paratraqueal son fáciles de distinguir por el contraste con las fibras. Asimismo, resalta el uso práctico que representa la evaluación macroscópica del carbón a través de patrones disponibles para los supervisores forestales, que normalmente usan lupas cuyo aumento no permite llegar a muchos detalles debiendo enfocarse en los elementos xilemáticos de la sección transversal.

Nigoski et al. (2012), reportaron en la madera y carbón de Dipteryx odorata características anatómicas como porosidad difusa, vasos predominantemente solitarios y escasos múltiples radiales, parcialmente obstruidos, parénquima axial aliforme y confluente y radios finos. Las pequeñas dimensiones celulares impidieron la visualización del leño carbonizado con menores aumentos, siendo detallados con el microscopio electrónico de barrido. Comparando imágenes de madera y carbón, la muestra de carbón presentó contracción tangencial de diámetro de poros, distinción de fibras y parénquima axial como consecuencia del distinto espesor de pared celular en el plano transversal; contracción mayor de radios uniseriados y estratificados en altura y ancho aumentando visualmente la frecuencia en el plano tangencial; presencia de puntuaciones intervasculaes alternas y los cristales en células de parénquima radial presentaron pequeñas grietas causadas por la acción de la temperatura de carbonización..

Resultado similar reportó Albuquerque (2012), en la evaluación del carbón de Dipteryx odorata, señalando que a causa de la carbonización los elementos xilemáticos presentaron variaciones dimensionales, como contracción del diámetro tangencial, longitud de vasos y altura y ancho de radios. Asimismo, para el carbón de Copaifera sp. reportó que el elemento xilemático con mayor contracción volumétrica producto de la carbonización a $400^{\circ} \mathrm{C}$ fue la longitud de vasos, seguido del diámetro de 
vasos y altura de radios. No obstante, ambas especies pudieron ser diferenciados e identificadas.

Las propiedades del carbón vegetal están directamente relacionadas con las características físicas, químicas y anatómicas de la madera utilizada en la carbonización y dentro de las propiedades físicas, la densidad básica es la de mayor influencia en la calidad del carbón vegetal (Trugilho y Silva 2001). Para carbón de Eucalyptus urophylla S.T. Blake y Corymbia citriodora (Hook.) K.D Hill \& L.A.S. Johnson, se indica que la temperatura es un factor importante en la calidad del carbón y en la composición química, puesto que a mayor temperatura final de carbonización, mayor será la cantidad de material volátil liberado. Asimismo, procesos de carbonización que utilizan temperaturas altas darán origen a un carbón con menor contenido de compuestos volátiles y mayores contenidos de carbono fijo y minerales (Couto et al. 2015).

El presente estudio tiene como objetivo caracterizar la estructura anatómica de la madera y el carbón de las especies capirona (Calycophyllum spruceanum), copaiba (Copaifera officinalis), shihuahuaco (Dipteryx micrantha), mango (Mangifera indica) y naranjo (Citrus sinensis), identificando las características anatómicas que se conservan después del proceso de carbonización. Además, evaluar las diferentes propiedades físicas y químicas del carbón que permitan diferenciarla de otros tipos de carbón. Es importante mencionar que dichas especies fueron elegidas por ser después del algarrobo los carbones más comercializados en el mercado local; asimismo, se tomó como base información bibliográfica referente a las características anatómicas y procesos de carbonización de especies comerciales de otros países, debido a la ausencia de investigación en caracterización anatómica de carbón en Perú.

\section{Materiales y Métodos}

El estudio anatómico de la madera y el carbón se realizó en el Laboratorio de Anatomía de la Madera, la carbonización y análisis químico del carbón en el Laboratorio de Trans- formación Química de la Madera; ambos laboratorios del Departamento de Industrias Forestales de la Facultad de Ciencias Forestales de la Universidad Nacional Agraria La Molina (Lima, Perú). Las microfotografías de fragmentos de carbón se obtuvieron en el Laboratorio de Equipamiento Especializado de la Unidad de Posgrado de la Facultad de Ciencias Biológicas de la Universidad Nacional Mayor de San Marcos (Lima, Perú).

\section{Metodología para madera}

Las muestras de madera de capirona (Calycophyllum spruceanum, Rubiaceae) fueron proporcionadas por el Laboratorio de Anatomía de la Madera, al igual que las de copaiba (Copaifera officinalis) y shihuahuaco (Dipteryx micrantha), ambas de la familia Fabaceae, en tanto que el mango (Mangifera indica, Anacardiaceae) proviene de la ciudad de Pucallpa (Ucayali) y el naranjo (Citrus sinensis, Rutaceae) del campus de la Universidad Nacional Agraria La Molina.

Para la descripción de la madera de las cinco especies se utilizó la Norma de procedimientos en estudios de anatomía de Madeira: I. Angiospermae del IBAMA (1992) y la List of microscopic features for hardwood identification (IAWA, 1989). La descripción de las características generales y macroscópicas de la madera se llevó a cabo con diez muestras (cantidad aprobada estadísticamente) de madera de $3 \times 3 \times 5 \mathrm{~cm}$ por especie, las mismas que estuvieron debidamente orientadas en sus tres secciones de corte (transversal, tangencial y radial). Mientras que para la caracterización microscópica se preparó veinte láminas de tejido macerado y veinte láminas histológicas de sus tres secciones transversal, tangencial y radial, que permitieron describir y medir los elementos leñosos mediante el software LAS de LEICA. Los parámetros estadísticos de los elementos anatómicos se calcularon conforme a lo indicado en la norma COPANT N³0 (1972).

\section{Metodología para carbón}

La carbonización se realizó con las mismas diez probetas por especie de $3 \times 3 \times 5 \mathrm{~cm}$ utilizadas para la caracterización anatómica de 
Caracterización anatómica de la madera y carbón de cinco especies leñosas para su identificación

la madera. Con ayuda de una cuchilla se cortó parte de la sección transversal de la probeta que luego fue marcada con lápiz y codificada de manera que después del proceso de carbonización se pudiera identificar. La carbonización se realizó en una mufla a una temperatura máxima de $450^{\circ} \mathrm{C}$ por el periodo de una hora. De las muestras carbonizadas y con ayuda de un estereoscopio se obtuvieron macrofotografías de las secciones antes demarcadas con lápiz, para realizar la comparación entre la madera y carbón que permita caracterizar e identificar la especie basándose en la estructura anatómica. Para cada especie de carbón se realizaron cincuenta mediciones del diámetro tangencial de poros y frecuencia de poros $/ \mathrm{mm}^{2}$ con el software Image Pro Plus v4.5. Los parámetros estadísticos de las características anatómicas evaluadas se calcularon según la norma COPANT N 30 (1972).

La evaluación físico-química del carbón vegetal se realizó con las muestras de las cinco especies, las cuales fueron sometidas a pruebas para determinar la densidad, contenido de humedad, cenizas, material volátil y carbono fijo según la Norma ASTM - D1762 (1984).

Cabe recalcar que al inicio del estudio no estuvo contemplado el uso de un microscopio electrónico de barrido; no obstante, durante el desarrollo de la investigación se presentó la oportunidad para analizar dos muestras de carbón, priorizando las especies capirona y shihuahuaco por ser las especies duras más utilizadas para hacer carbón en el ámbito nacional.

Finalmente, para el análisis de microscopía electrónica de barrido (SEM) se tomó parte del carbón molido de las especies capirona y shihuahuaco retenido en la malla del tamiz $\mathrm{N}^{\circ} 20(850 \mu \mathrm{m})$, es decir fragmentos de 850 a $1500 \mu \mathrm{m}$, los cuales fueron montados en porta-muestras cilíndricos de acero de $1 \mathrm{~cm}$ de diámetro y $0,5 \mathrm{~cm}$ de altura pegados con adhesivo sintético no líquido, para evitar su ascenso por capilaridad en el carbón y luego metalizados con oro para su observación. El proceso de preparación de las muestras y captura de microfotografías con el microscopio SEM Inspect S50 duró aproximadamente una hora.

\section{Resultados}

\section{Análisis a nivel madera}

A nivel macroscópico, el parénquima axial de capirona no fue visible; en copaiba fue vasicéntrico y marginal; en shihuahuaco vasicéntrico, aliforme y aliforme confluente; en mango vasicéntrico y aliforme, mientras que el naranjo además de presentar parénquima vasicéntrico, escaso aliforme y aliforme confluente presentó bandas del tipo marginal (Figura 1). Copaiba y shihuahuaco, presentaron gomas y sólo se observó sílice en los elementos vasculares de la especie shihuahuaco (Cuadro 1).

A nivel microscópico, capirona y naranjo presentaron apéndices en sus elementos vasculares en uno o ambos extremos (Figura 2). Solo la especie copaiba presentó canales intercelulares. Con respecto al parénquima, se observaron los mismos tipos que a nivel macroscópico. En cuanto a los radios, las especies shihuahuaco y naranjo presentaron radios homocelulares conformados por células procumbentes; mientras que el resto de especies presentaron radios heterocelulares del tipo II (Figura 3). Además, shihuahuaco fue la única especie que presentó radios estratificados y capirona radios fusionados (Cuadro 2).

La especie capirona presentó fibras largas de $1649 \mu \mathrm{m}$, el mango fibras cortas de $658 \mu \mathrm{m}$ y las otras tres especies fibras de longitud mediana de 736 a $1235 \mu \mathrm{m}$. El espesor de pared de las fibras con $5.3 \mu \mathrm{m}$ fue muy grueso en shihuahuaco; de 1.5 - $3.1 \mu \mathrm{m}$ muy delgadas en mango y para las otras especies de $2.0-3.5 \mu \mathrm{m}$ fue delgado; asimismo, el mango fue la única especie que presentó fibras septadas.

Como inclusiones se observaron gomas en copaiba, shihuahuaco, mango y naranjo. Además, copaiba y naranjo presentaron cristales de forma romboide, en el parénquima longitudinal y radios respectivamente. Las especies mango y naranjo presentaron tilosis; no obstante, esta última con paredes engrosadas.

\section{Análisis a nivel carbón vegetal}

En cuanto a las características macroscópicas del carbón, se verifica que las especies conser- 


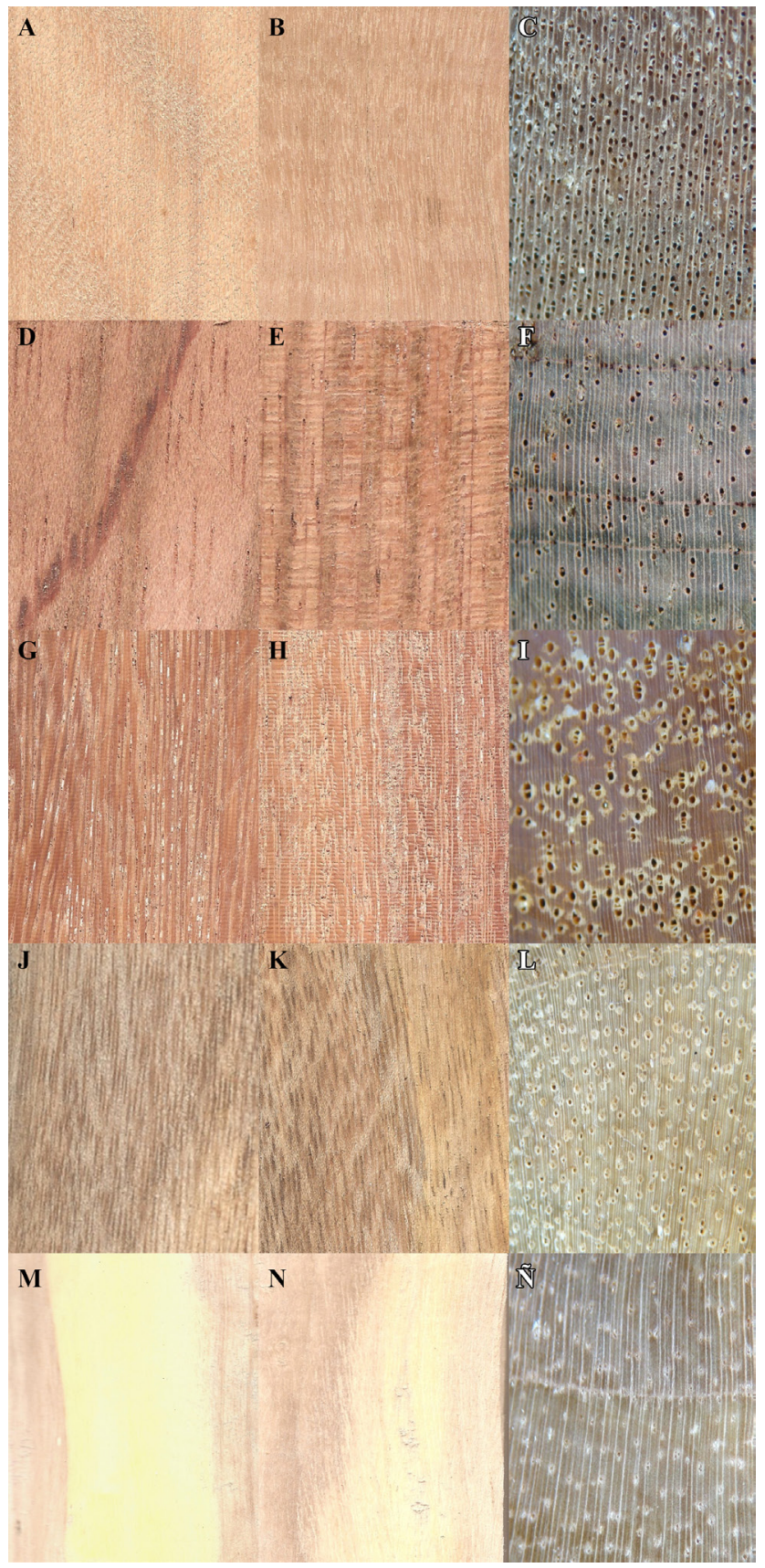

Figura 1. Secciones tangenciales de madera de A) capirona, D) copaíba, G) shihuahuaco, J) mango y M) naranjo. Secciones radiales de madera de B) capirona, E) copaíba, H) shihuahuaco, K) mango y N) naranjo. Secciones transversales de madera de C) capirona, F) copaíba, I) shihuahuaco, L) mango y N) naranjo a un aumento de $10 \mathrm{x}$. 


\begin{tabular}{|c|c|c|c|c|c|c|c|c|}
\hline $\begin{array}{l}Z Z \\
\text { 营. } \\
\text { E. }\end{array}$ & $\underset{0}{3}$ & $\begin{array}{l}\text { D. } \\
\text { E. } \\
\text { (2) }\end{array}$ & 尺. & 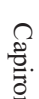 & \multicolumn{4}{|c|}{ ESPECIES } \\
\hline & & & $x$ & & Rojo & \multirow{4}{*}{\multicolumn{2}{|c|}{ Color }} & \multirow{16}{*}{ 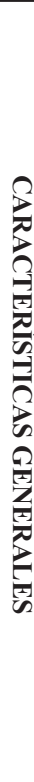 } \\
\hline$x$ & $x$ & & & & Amarillo & & & \\
\hline & & & & $x$ & Pardo & & & \\
\hline & & $x$ & & & Marrón & & & \\
\hline & & & $x$ & $x$ & Recto & \multirow{2}{*}{\multicolumn{2}{|c|}{ Grano }} & \\
\hline$x$ & $x$ & $x$ & & $x$ & Entrecruzado & & & \\
\hline$x$ & & & & $x$ & Fina & \multirow{2}{*}{\multicolumn{2}{|c|}{ Textura }} & \\
\hline & $x$ & $x$ & $x$ & & Media & & & \\
\hline$x$ & & & & $x$ & Ausente & \multirow{2}{*}{\multicolumn{2}{|c|}{ Brillo }} & \\
\hline & $x$ & $x$ & $x$ & & Medio & & & \\
\hline & & $x$ & $x$ & & Arcos superpuestos & \multirow{4}{*}{\multicolumn{2}{|c|}{ Veteado }} & \\
\hline & & $x$ & & & Satinado & & & \\
\hline$x$ & & & & & Característicos & & & \\
\hline & $x$ & & & $x$ & Ausente & & & \\
\hline & $x$ & & & & Blanda & \multirow{2}{*}{\multicolumn{2}{|c|}{ Resistencia manual al corte }} & \\
\hline$x$ & & $x$ & $x$ & $x$ & Dura & & & \\
\hline$x$ & & & & $x$ & No visible a simple vista & \multirow{2}{*}{ Visibilidad } & \multirow{4}{*}{ Poros } & \\
\hline & $x$ & $x$ & $x$ & & Ligeramente visible a simple vista & & & \\
\hline$x$ & $x$ & 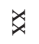 & $\underset{x}{x}$ & $x$ & Solitarios & \multirow{2}{*}{ Agrupamiento } & & $\Omega$ \\
\hline$x$ & $x$ & $x$ & $x$ & $x$ & Multiples & & & $\sum^{2}$ \\
\hline & & & $x$ & & \multicolumn{3}{|c|}{ Canales intercelulares } & 胥 \\
\hline & & & & $x$ & \multicolumn{2}{|c|}{ No visible aún con lupa de $10 x$} & \multirow{6}{*}{ Parénquima } & $\vec{\sigma}$ \\
\hline$x$ & $x$ & $x$ & $x$ & & \multicolumn{2}{|c|}{ Visible con lupa de 10x } & & $\bar{\Omega}$ \\
\hline$x$ & $x$ & $x$ & $x$ & & Vasicéntrico & \multirow{3}{*}{ Paratraqueal } & & 7 \\
\hline$x$ & $x$ & $x$ & & & Aliforme & & & 8 \\
\hline$x$ & & $x$ & & & Aliforme confluente & & & 0 \\
\hline$x$ & & & $x$ & & Marginal & En bandas & & 2. \\
\hline$x$ & $x$ & $x$ & $x$ & $x$ & \multicolumn{2}{|c|}{ Visibles con lupa de $10 x$} & \multirow{2}{*}{ Radios } & $\bar{\Omega}$ \\
\hline & & $x$ & & & \multicolumn{2}{|l|}{ Estratificados } & & is \\
\hline & & $x$ & $x$ & & \multicolumn{2}{|l|}{ Gomas } & \multirow{2}{*}{ Otros } & \\
\hline & & $x$ & & & \multicolumn{2}{|l|}{ Sílice } & & \\
\hline
\end{tabular}

Cuadro 1. Características generales y macroscópicas de la madera de las cinco especies en estudio (xx: Predomina). 

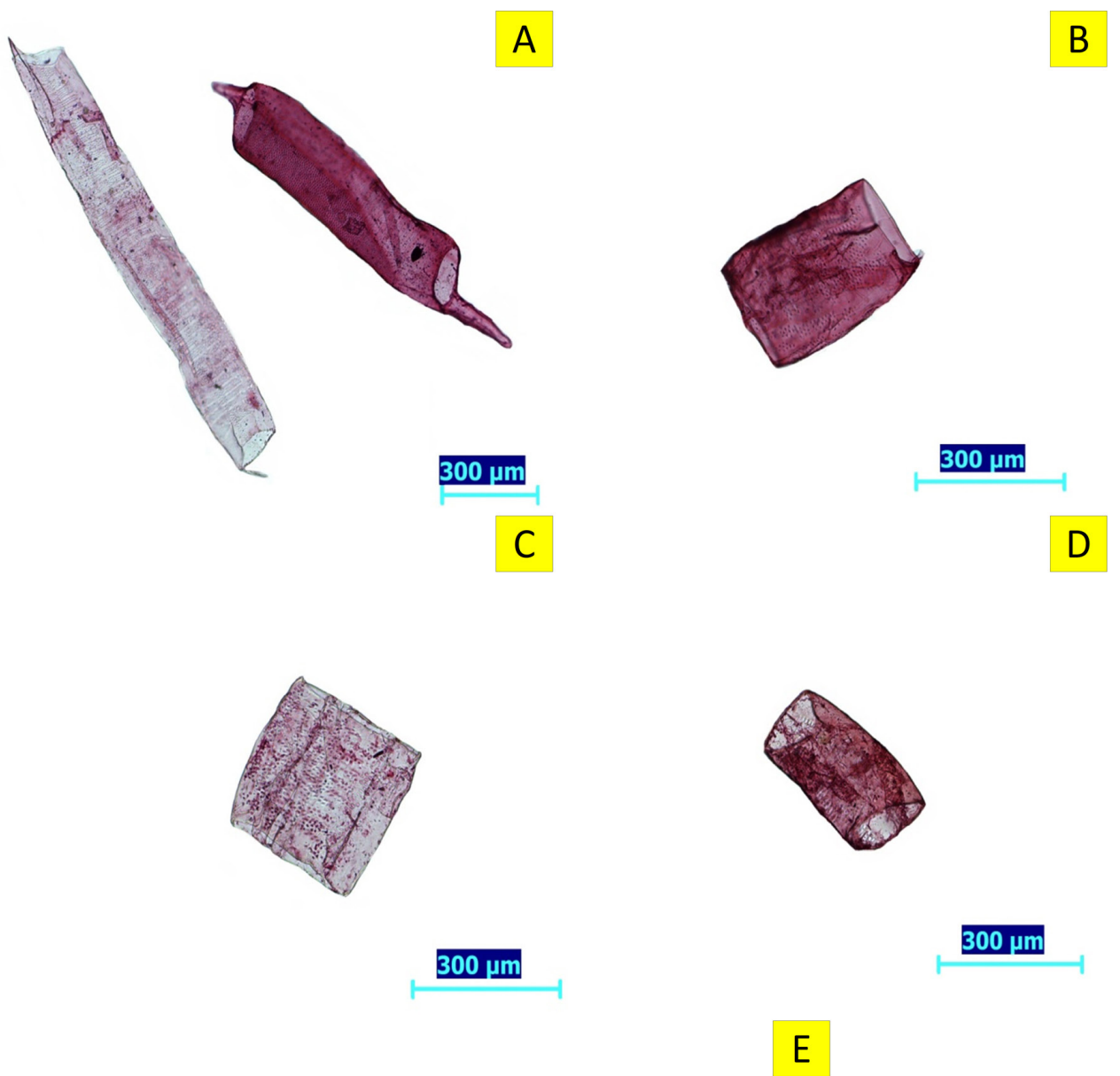

$300 \mathrm{um}$

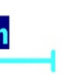

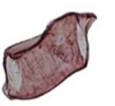

\section{$300 \mu \mathrm{m}$}

Figura 2. Elementos vasculares de la madera de A) capirona, B) copaíba, C) shihuahuaco, D) mango y E) naranjo a un aumento de 10x. 
Caracterización anatómica de la madera y carbón de cinco

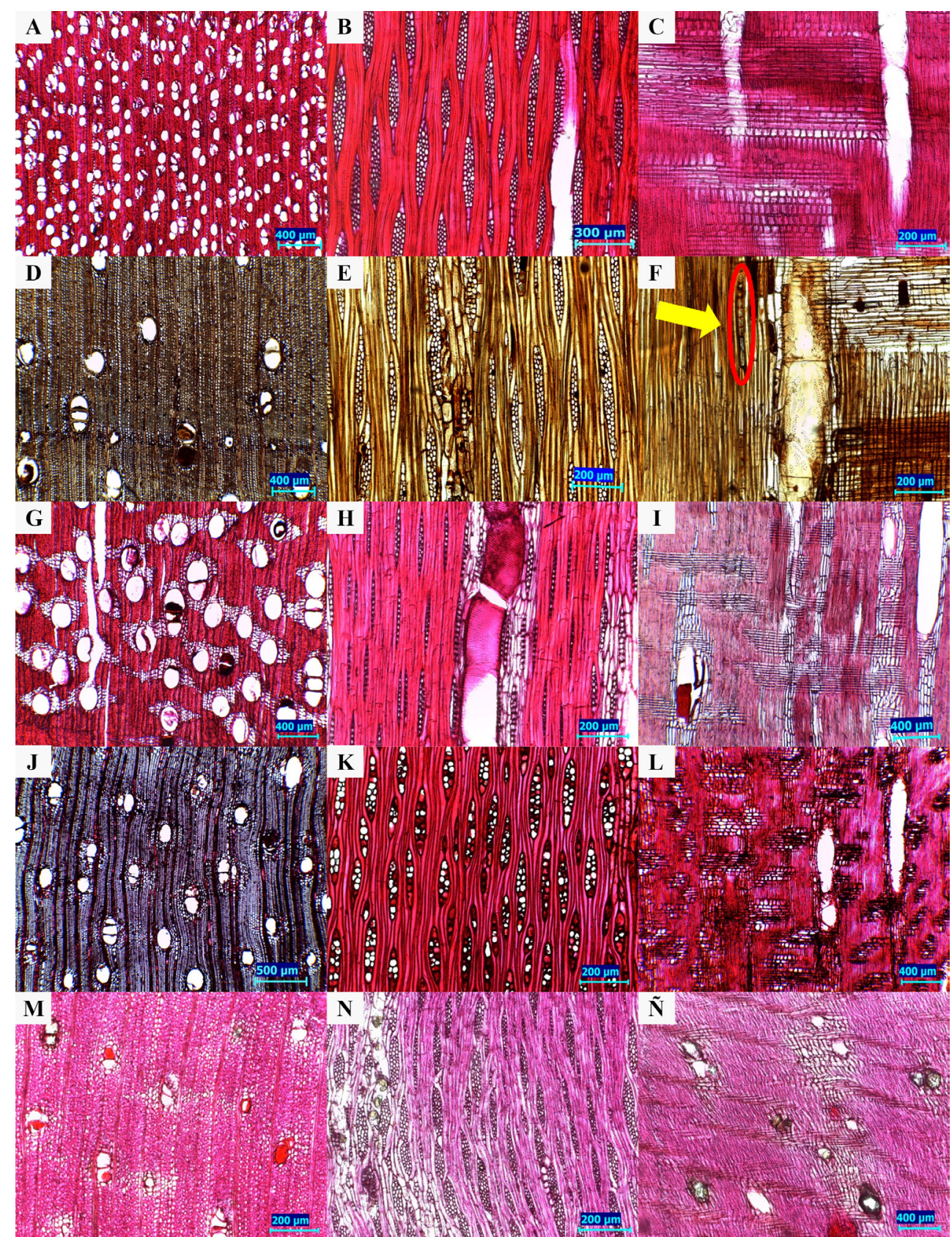

Figura 3. Secciones transversales a nível microscópico de la madera de A) capirona, D) copaíba, G) shihuahuaco, J) mango y M) naranjo a un aumento de 4x. Secciones tangenciales a nível microscópico de la madera de B) capirona, E) copaíba, H) shihuahuaco, K) mango con fibras septadas y N) naranjo a un aumento de 10x. Secciones radiales a nível microscópico de la madera de C) capirona, F) copaíba, con cristales cuadrangulares en el parénquima longitudinal a un aumento de $10 \mathrm{x}$ y I) shihuahuaco L) mango y Ñ) naranjo a un aumento de $4 \mathrm{x}$. 


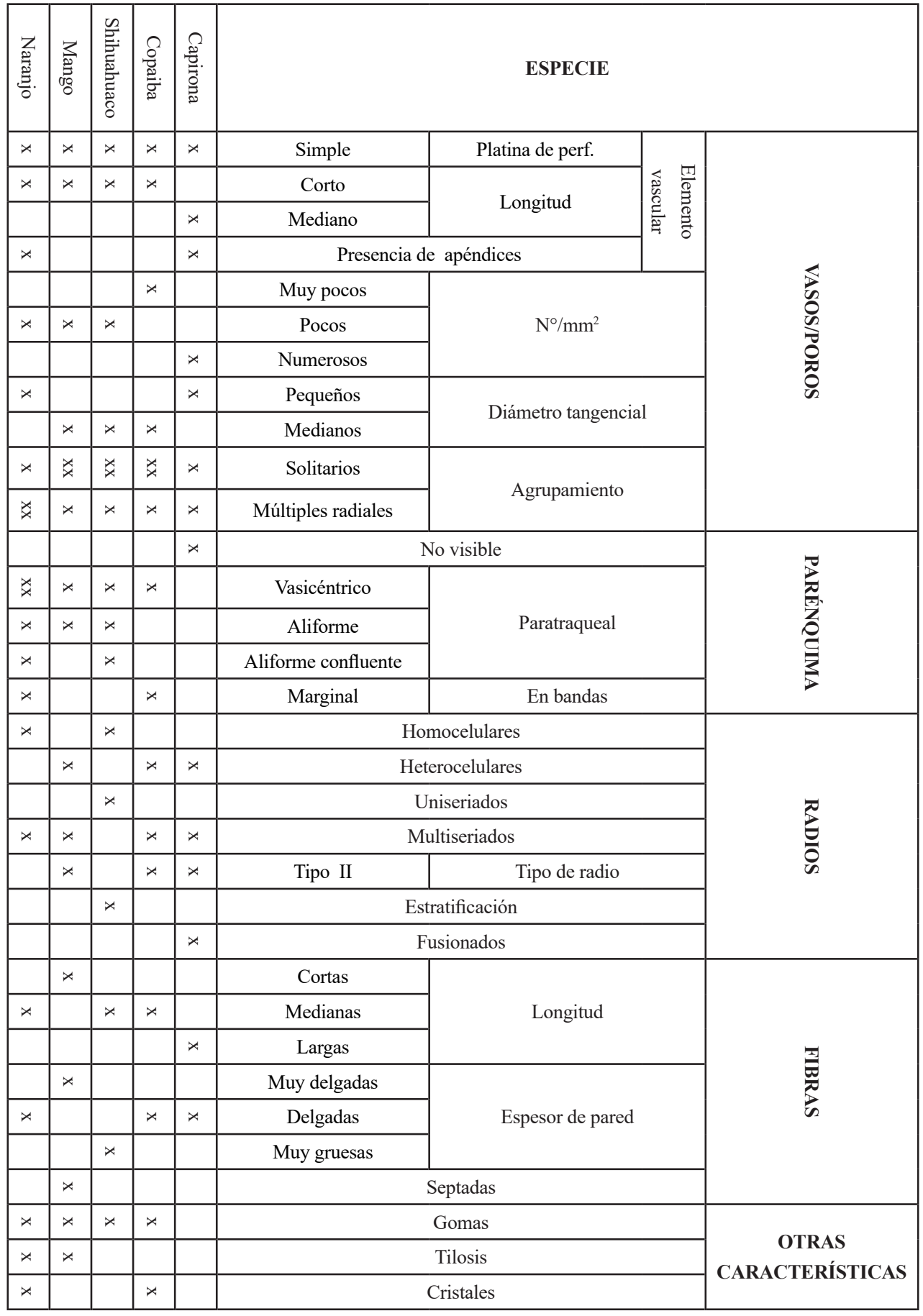

Cuadro 2. Características microscópicas de la madera de las cinco especies en estudio (xx: Predomina). 
van su agrupamiento de poros, conforme a la descripción anatómica de la madera (Cuadro 3). Sin embargo, en todos los casos el diámetro tangencial de poros en carbón fue menor producto de la contracción de la madera, ocasionando un aumento en la densidad de poros por $\mathrm{mm}^{2}$ (Cuadro 4). Cabe resaltar que el carbón de mango presentó la mayor contracción de diámetro de poros.

Con respecto al parénquima axial, en la especie capirona no llegó a observarse y en copaiba perdura el parénquima marginal, inclusive se conservan los canales gomíferos. No obstante, el parénquima vasicéntrico no llega a visualizarse. En shihuahuaco se conservó el parénquima vasicéntrico, aliforme y aliforme confluente; en mango el parénquima vasicéntrico y aliforme perduraron, notándose abundante tejido parenquimático alrededor de los poros. En naranjo el parénquima vasicéntrico, aliforme, aliforme confluente y marginal se llegó a observar, pero con dificultad (Figura 4).

El carbón de capirona presentó mayor densidad. El contenido de humedad obtenido para todas las especies osciló entre 5-7 por ciento. El material volátil presenta valores por encima del 30 por ciento; para el caso del carbón de naranjo presenta un valor elevado de material volátil, probablemente relacionado con componentes propios de su madera. Y el contenido de cenizas fue variable en las cinco especies estudiadas (Cuadro 5).

\section{Análisis de carbón molido al microscopio electrónico de barrido}

Este análisis se realizó para fragmentos de carbón de capirona y shihuahuaco, permitiendo en algunos casos visualizar la estructura difícil de observar con el estereoscopio. En la muestra de capirona se pueden observar los radios multiseriados en la sección tangencial (Figura 5A y 5B) mientras que en un fragmento de la sección transversal se notan poros rodeados por numerosas y pequeñas fibras, pero no se evidencia el parénquima (Figura 5C), el cual tampoco se visualiza en su madera. Además en la Figura 5D, se observa un elemento vascular con punteaduras intervasculares las cuales se pudieron apreciar con mayor facilidad a un aumento de 6000x (Figura 5E). En shihuahuaco en la sección tangencial se pudieron observar dos líneas horizontales de células que constituyen dos radios uniseriados (Figura 6A y 6B).

\section{Discusión}

\section{Características anatómicas de la madera}

En la observación macroscópica de la madera de capirona el parénquima no fue visible, resultado que concuerda con Chavesta (2005). Nuestro resultado para copaiba coincide con Acevedo y Kikata (1994), ya que se visualizó el mismo tipo de parénquima axial, radios e inclusiones; para shihuahuaco, además del parénquima vasicéntrico y aliforme expuesto por Chavesta (2015), se observó el aliforme confluente. Es importante resaltar que el autor Chavesta considera como parte de su descripción anatómica a la especie Dipteryx odorota; no obstante, recientes estudios realizados por Honorio et al. (2018) y Aldana et al. (2016), demuestran que la especie que se distribuye ecológicamente en Perú es Dipteryx micrantha y no Dipteryx odorata como se estuvo reportando en investigaciones pasadas, por lo que todos los estudios realizados en Perú que hacen referencia a esta última, en realidad se refieren a Dipteryx micrantha. La presencia de gomas en copaiba y shihuahuaco coincide con lo reportado por Acevedo y Kikata (1994) y Chavesta (2015), respectivamente.

A nivel microscópico, se observó canales intercelulares en copaiba tal y como fue reportado por Acevedo y Kikata (1994). Respecto al parénquima, la no visibilidad de esta característica en capirona coincide con Chavesta (2005); mientras que la presencia de paratraqueal vasicéntrico y bandas del tipo marginal en copaiba coincide con lo reportado por Acevedo y Kikata (1994); los tipos de parénquima que presentó shihuahuaco están acordes con lo encontrado por Chavesta (2015); en tanto que el mango está conforme con lo expuesto por León (2014) y lo reportado para el naranjo con Brenes et al. (2012).

\section{Características anatómicas del carbón vege- tal}

En el análisis comparativo entre la estructura anatómica de la madera y carbón, los resulta- 


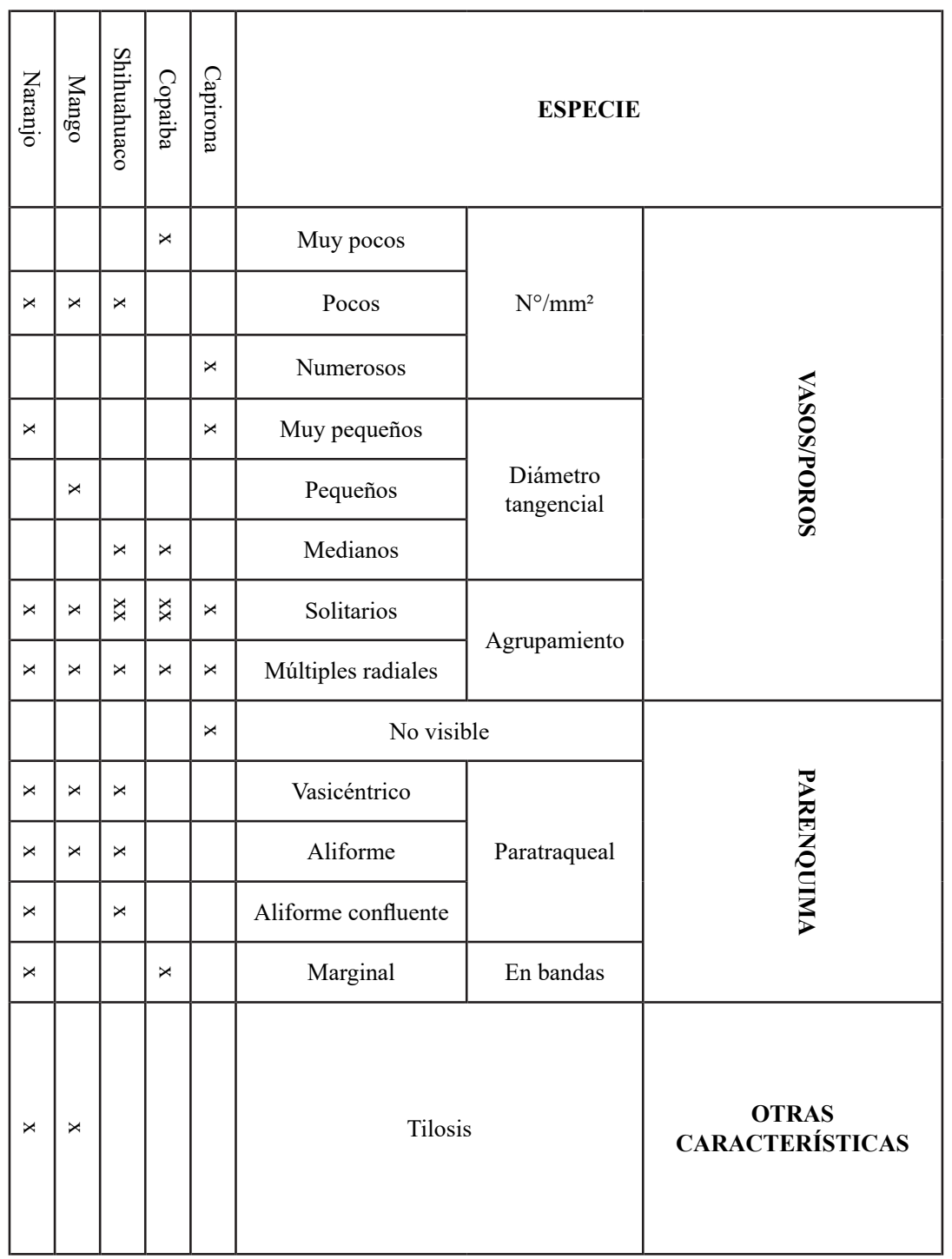

Cuadro 3. Características macroscópicas del carbón de las cinco especies en estudio (xx: Predomina).

dos obtenidos corroboran lo reportado por Gonçalves (2010, 2015); Muñiz et al. (2012a); Nigoski et al. (2012) y Albuquerque (2012); para las especies capirona, copaiba y shihuahuaco (género Dipteryx sp.).

En el carbón de copaiba el parénquima vasicéntrico no llega a visualizarse, debido a la contracción y poca cantidad de células alrede- dor del poro, coincidiendo con Muñiz et al. (2012a) quien señala que en un mismo proceso de carbonización el comportamiento dimensional de las estructuras anatómicas es distinto, influenciado muchas veces por la cantidad de elementos celulares. En naranjo el parénquima se observó con dificultad, a pesar de tener vasicéntrico, aliforme, aliforme confluente 


\begin{tabular}{|c|c|c|c|c|}
\hline \multirow{3}{*}{ Concepto } & \multicolumn{2}{|c|}{ Madera } & \multicolumn{2}{|c|}{ Carbón } \\
\hline & \multicolumn{2}{|c|}{ Poros } & \multicolumn{2}{|c|}{ Poros } \\
\hline & $\begin{array}{c}\text { Diámetro } \\
\text { tangencial }\end{array}$ & $\begin{array}{c}\text { Número por } \\
\mathbf{m m}^{2}\end{array}$ & $\begin{array}{l}\text { Diámetro tan- } \\
\text { gencial }\end{array}$ & $\begin{array}{c}\text { Número por } \\
\mathbf{m m}^{2}\end{array}$ \\
\hline \multicolumn{5}{|c|}{ Capirona } \\
\hline Valor Promedio & 59 & 43 & 42 & 48 \\
\hline Valor mínimo & 48 & 30 & 27 & 34 \\
\hline Valor máximo & 90 & 59 & 59 & 61 \\
\hline Desviación estándar & 9 & 5 & 8 & 5 \\
\hline Coeficiente de variabilidad & 14 & 12 & 19 & 11 \\
\hline Intervalo de confianza & $59 \pm 2$ & $43 \pm 1$ & $42 \pm 2$ & $48 \pm 2$ \\
\hline \multicolumn{5}{|c|}{ Copaiba } \\
\hline Valor Promedio & 152 & 4 & 123 & 4 \\
\hline Valor mínimo & 81 & 2 & 85 & 3 \\
\hline Valor máximo & 212 & 7 & 185 & 7 \\
\hline Desviación estándar & 24 & 1 & 25 & 1 \\
\hline Coeficiente de variabilidad & 16 & 31 & 20 & 26 \\
\hline Intervalo de confianza & $152 \pm 7$ & $4 \pm 1$ & $123 \pm 7$ & $4 \pm 0$ \\
\hline \multicolumn{5}{|c|}{ Shihuahuaco } \\
\hline Valor Promedio & 164 & 6 & 136 & 10 \\
\hline Valor mínimo & 116 & 4 & 88 & 7 \\
\hline Valor máximo & 202 & 11 & 193 & 13 \\
\hline Desviación estándar & 19 & 2 & 24 & 2 \\
\hline Coeficiente de variabilidad & 12 & 24 & 18 & 17 \\
\hline Intervalo de confianza & $164 \pm 5$ & $6 \pm 1$ & $136 \pm 7$ & $10 \pm 0$ \\
\hline \multicolumn{5}{|c|}{ Mango } \\
\hline Valor Promedio & 129 & 5 & 78 & 7 \\
\hline Valor mínimo & 93 & 3 & 43 & 4 \\
\hline Valor máximo & 168 & 8 & 137 & 9 \\
\hline Desviación estándar & 16 & 1 & 18 & 1 \\
\hline Coeficiente de variabilidad & 12 & 21 & 23 & 18 \\
\hline Intervalo de confianza & $129 \pm 5$ & $5 \pm 0$ & $78 \pm 5$ & $7 \pm 0$ \\
\hline \multicolumn{5}{|c|}{ Naranjo } \\
\hline Valor Promedio & 53 & 13 & 35 & 19 \\
\hline Valor mínimo & 32 & 7 & 22 & 11 \\
\hline Valor máximo & 68 & 19 & 67 & 26 \\
\hline Desviación estándar & 8 & 3 & 8 & 3 \\
\hline Coeficiente de variabilidad & 15 & 22 & 24 & 18 \\
\hline Intervalo de confianza & $53 \pm 3$ & $13 \pm 1$ & $35 \pm 2$ & $19 \pm 1$ \\
\hline
\end{tabular}


Cuadro 4. Valores de diámetro y frecuencia de poros/mm2 en madera y carbón (en página anterior).

\begin{tabular}{|c|c|c|c|c|c|}
\hline Características & Capirona & Copaiba & Shihuahuaco & Mango & Naranjo \\
\hline $\begin{array}{c}\text { Densidad de la } \\
\text { Madera }\end{array}$ & 0,76 & 0,61 & 0,87 & $0,52-0,7$ & $0,49-0,78$ \\
\hline \multicolumn{6}{|c|}{ Densidad de carbón } \\
\hline Promedio & 0,53 & 0,30 & 0,48 & 0,28 & 0,46 \\
\hline Desv. standard & 0,04 & 0,03 & 0,02 & 0,03 & 0,04 \\
\hline $\mathrm{CV} \%$ & 7,30 & 9,20 & 3,20 & 10,06 & 8,67 \\
\hline \multicolumn{6}{|c|}{ Contenido de humedad (\%) } \\
\hline Promedio & 5,42 & 5,74 & 5,83 & 7,00 & 5,45 \\
\hline Desv. standard & 0,59 & 0,28 & 0,11 & 0,61 & 0,76 \\
\hline $\mathrm{CV} \%$ & 10,91 & 4,89 & 1,85 & 8,66 & 14,00 \\
\hline \multicolumn{6}{|c|}{ Material volátil (\%) } \\
\hline Promedio & 36,50 & 41,17 & 32,94 & 32,02 & 50,36 \\
\hline Desv. standard & 1,67 & 4,63 & 0,62 & 0,43 & 3,14 \\
\hline $\mathrm{CV} \%$ & 4,59 & 11,25 & 1,87 & 1,35 & 6,23 \\
\hline \multicolumn{6}{|c|}{ Ceniza (\%) } \\
\hline Promedio & 2,20 & 2,16 & 1,34 & 2,81 & 2,68 \\
\hline Desv. standard & 0,16 & 0,06 & 0,02 & 0,65 & 0,08 \\
\hline $\mathrm{CV} \%$ & 7,36 & 2,95 & 1,48 & 22,98 & 2,91 \\
\hline \multicolumn{6}{|c|}{ Carbono fijo (\%) } \\
\hline Promedio & 55,88 & 50,92 & 59,90 & 58,17 & 41,52 \\
\hline Desv. standard & 1,63 & 4,40 & 0,70 & 1,67 & 2,47 \\
\hline $\mathrm{CV} \%$ & 2,92 & 8,63 & 1,17 & 2,88 & 5,96 \\
\hline
\end{tabular}

Cuadro 5. Propiedades físicas y químicas del carbón de las especies estudiadas.

y bandas del tipo marginal en contraste a lo expuesto por Gonçalves et al. (2015), quienes argumentan que estos tipos de parénquimas por lo general son fáciles de diferenciarse por el contraste con las fibras.

Conforme a lo observado, los elementos xilemáticos de las cinco especies en estudio han diferido en su comportamiento dimensional bajo el mismo método de carbonización, debido a la variación en el espesor de la pared de fibras, cantidad de parénquima y ancho de radios; sin embargo, las contracciones no impi- dieron la identificación de la especie de acuerdo a lo reportado por Gonçalves (2010), Albuquerque (2012), Muñiz et al. (2013) y Nigoski et al. $(2012,2015)$.

El parénquima axial es considerado una de las principales características anatómicas para identificar una especie en madera y carbón, la cual se visualizó en las muestras de carbón de cuatro de las cinco especies en estudio, el tamaño y distribución de poros también son importantes coincidiendo con lo mencionado por Gonçalves et al. (2015). Asimismo, otras 
Caracterización anatómica de la madera y carbón de cinco

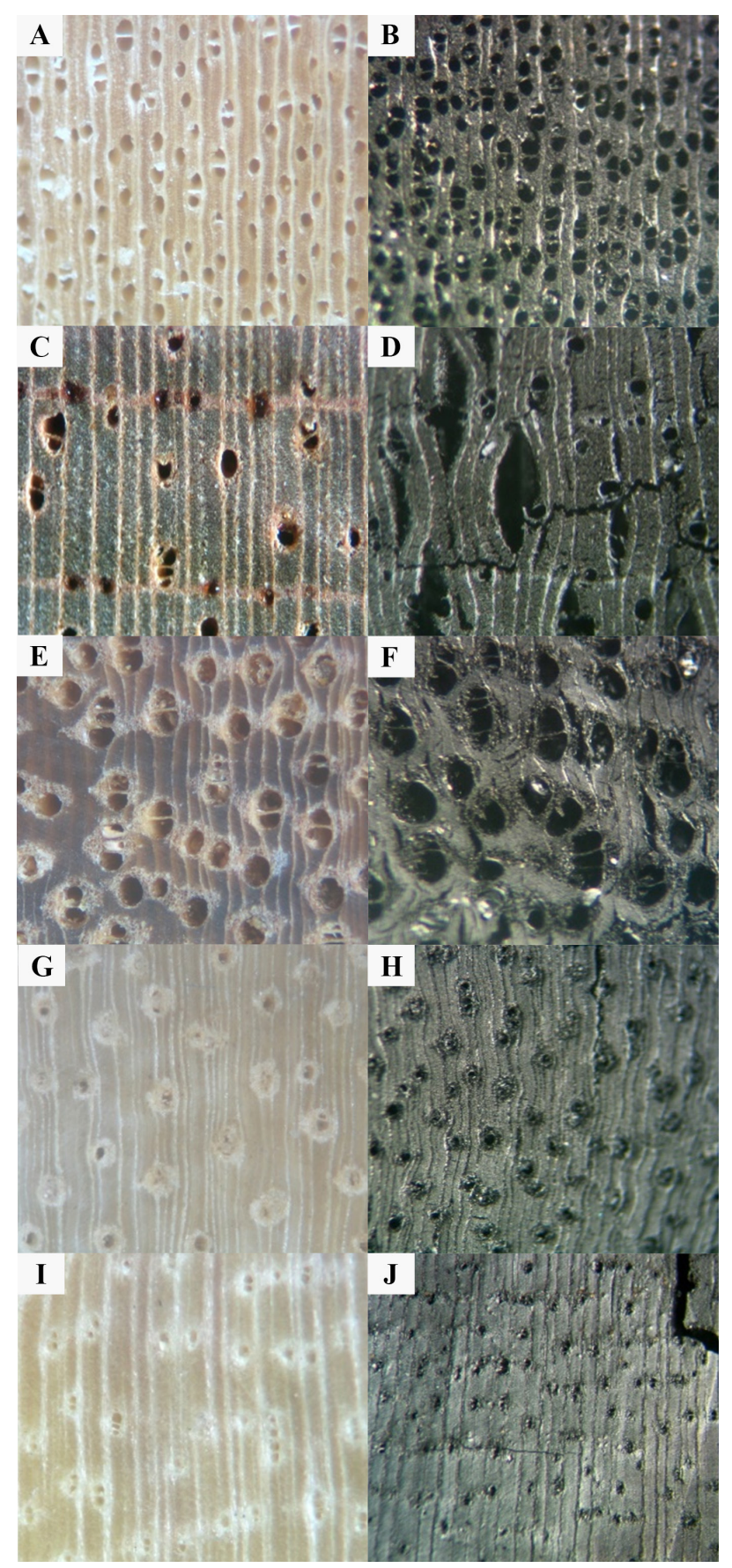

Figura 4. Secciones transversales de madera (a la izquierda) y carbón (a la derecha). A y B) capirona, C y D) copaiba, E y F) shihuahuaco, G y H) mango, I y J) naranjo a un aumento de 40x. 

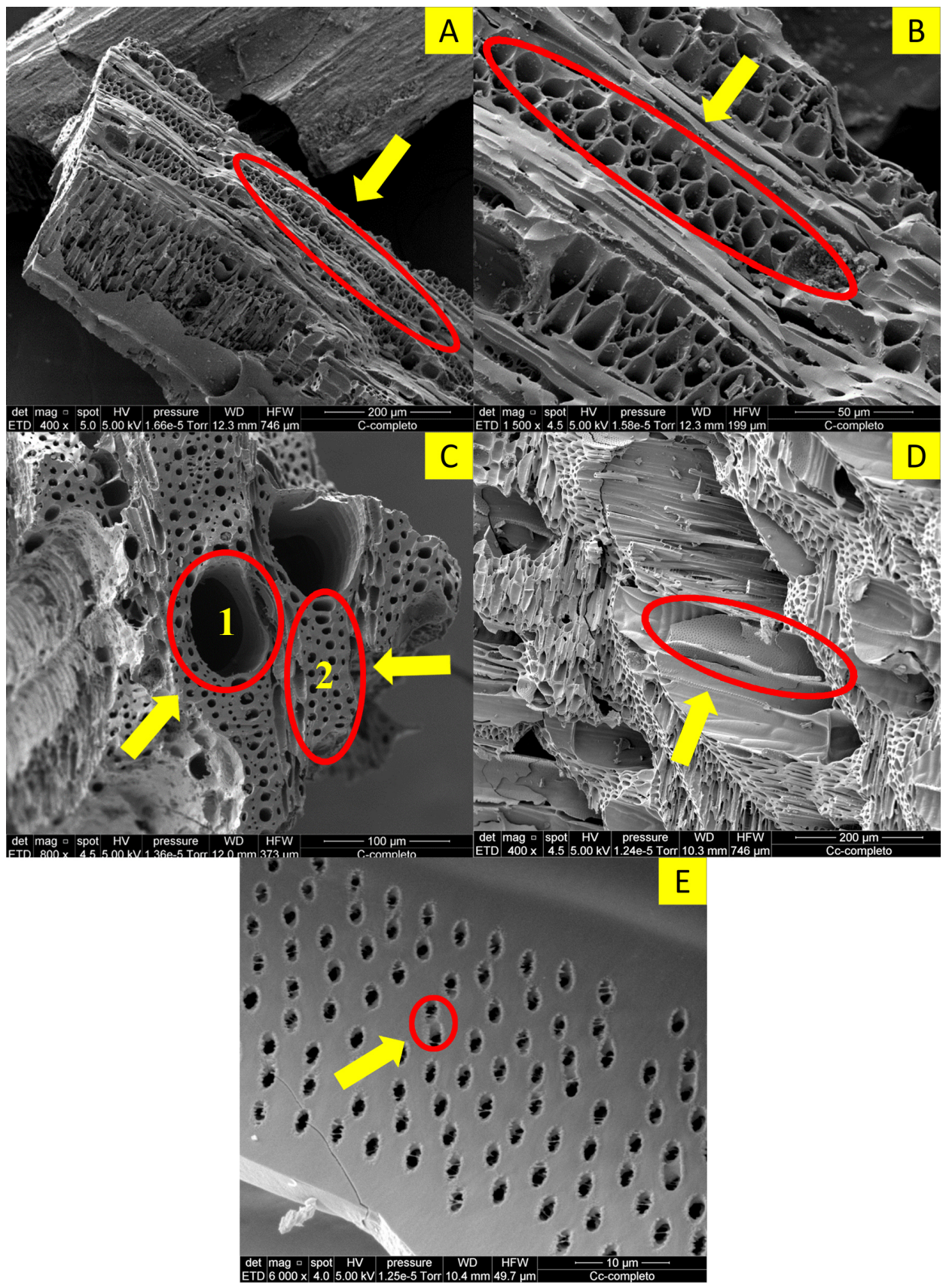

Figura 5. Imágenes de fragmentos de carbón de capirona con microscopio electrónico de barrido (SEM), A) radio multiseriado en sección tangencial a 400x, B) radio multiseriado en sección tangencial a 1500x, C1) poro en sección transversal a 800x, C2) fibras en sección transversal a 800x, D) elemento vascular con punteaduras a 400x y E) punteaduras intervascualres a 6000x. 


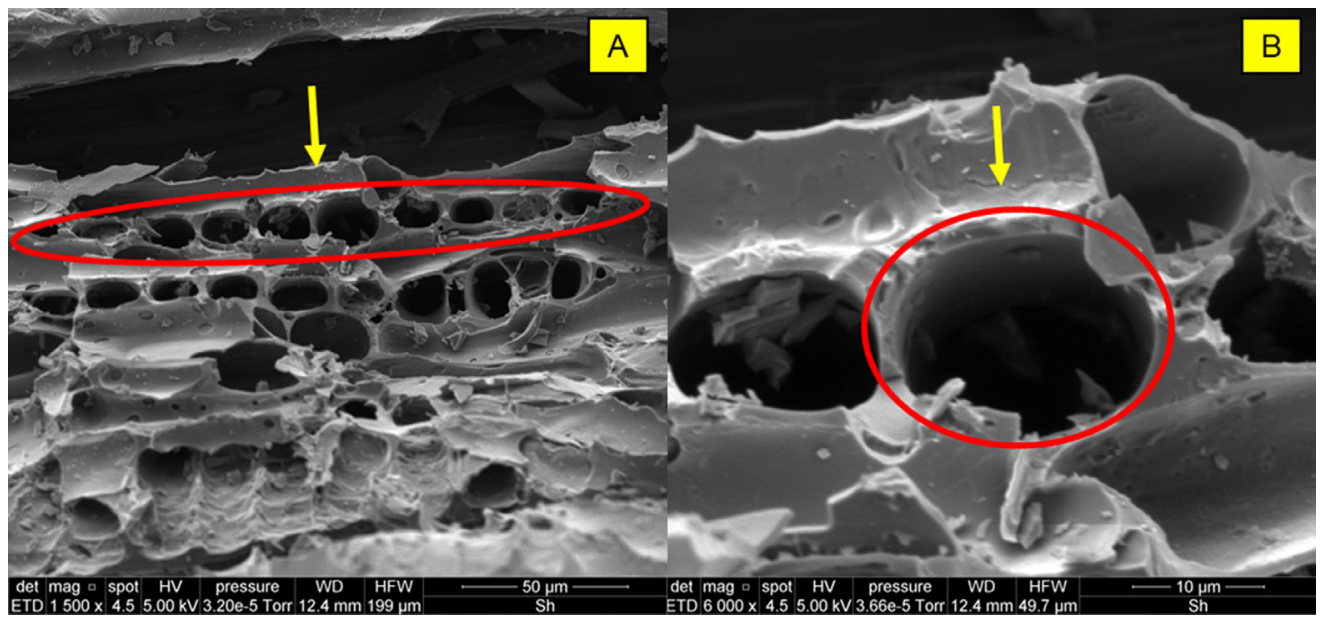

Figura 6. Imágenes de la sección tangencial de fragmentos de carbón de shihuahuaco con microscopio electrónico de barrido (SEM), A) radio uniseriado a 1500x y B) Célula de radio uniseriado a 6000x.

características importantes que se conservan después de la carbonización y contribuyen con el reconocimiento de las especies, son los canales gomíferos, los anillos de crecimiento y la tilosis; no obstante, la presencia de esta última característica difiere con lo reportado por Pipa et al. (2009).

\section{Propiedades físicas y químicas del carbón vegetal}

Conforme lo señala Earl (1975), Brito y Barrichelo (1980) y Trugilho y Silva (2001), se evidencia una relación directa entre la densidad básica de la madera con los valores de densidad aparente del carbón. Se esperaba que la densidad aparente del carbón de shihuahuaco fuese mayor al resto; sin embargo, el valor más alto fue para capirona; cuya posible causa sería la cantidad de material volátil presente, puesto que los residuos líquidos y alquitranados contribuyen en el peso del carbón y por ende en la densidad; por ello, el carbón de capirona al contener más material volátil supera en densidad aparente al carbón de shihuahuaco.

Existe una relación inversa entre los valores de material volátil con la cantidad de carbono fijo, lo cual es confirmado por Couto et al. (2015). La cantidad de material volátil depende del tiempo y temperatura de carbonización, así como de la composición de la madera.

Las especies capirona, copaiba y shihuahuaco presentaron una relación importante entre el contenido de carbono fijo y la densidad aparente, la que a su vez se correlaciona con la densidad de la madera. Dicha relación está influenciada en parte por el contenido de lignina, componente químico constituyente de la madera con estabilidad estructural capaz de soportar altas temperaturas y contribuir en la concentración del carbono fijo; incrementando así la densidad, conforme a lo mencionado por Brito y Barrichelo (1977).

\section{Conclusiones}

Los elementos leñosos se conservan después del proceso de carbonización permitiendo la identificación de las especies estudiadas, siendo el parénquima axial la principal característica anatómica que se mantiene, excepto para la especie copaiba donde no se visualizó el parénquima de tipo vasicéntrico.

Una de las características que permitirán diferenciar el carbón de la especie copaiba es la presencia de estructuras como los canales gomíferos, que se diferencian de los poros al 
ser más pequeños, mientras que para las especies mango y naranjo rasgos como la tilosis pueden ayudar a su identificación.

La reducción del tamaño de los poros e incremento de su densidad en número de poros/ $\mathrm{mm}^{2}$ durante el proceso de carbonización no impide la identificación de las especies forestales en estudio.

Sustancias químicas como las gomas y sílice no se conservan después del proceso de carbonización, por lo que no se consideran rasgos relevantes para la identificación anatómica del carbón.

La densidad del carbón de shihuahuaco no fue superior al resto de especies estudiadas, por lo que la madera que presenta mayor densidad no siempre produce un carbón más denso.

Las propiedades químicas como la cantidad de material volátil, que mantiene una relación inversamente proporcional con el contenido de carbono fijo del carbón, permite caracterizar el carbón de las especies estudiadas, diferenciándolas de otros tipos de carbones como el carbón activado, carbón de cáscara de coco, etc, más no son características que permitan la diferenciación entre especies.

\section{Bibliografía}

Acevedo, M; Kikata, Y. 1994. Atlas de maderas del Perú. Lima, PE. 202 p.

Albuquerque, A. 2012. Anatomia comparada do lenho e do carvão aplicada na identificação de 75 espécies da floresta Amazonica, no estado do Pará, Brasil. Tesis Mg. Piracicaba, BR. Universidad de Sao Paulo Escola Superior de Agricultura “Luiz de Queiroz". 249p.

Aldana, D; García-Dávila, C; Hidalgo, C; Flores, G; Del Castillo, D; Reynel, C; Pariente, E; Honorio, E. 2016. Análisis morfométrico de las especies de Dipteryx en la Amazonía peruana. Folia Amazónica 25(2):101-118.

ASTM. 1984. American Society for Testing and Materials. Standard test method for chemical analysis of wood charcoal. Annual Book of ASTM Standards. ES.
Bennett-Curry, A; Malhi, Y; Menton, M. 2013. Leakage effects in natural resource supply chains: a case study from the Peruvian commercial charcoal market. International Journal of Sustainable Development \& World Ecology 20(4):336-348.

Brenes-Angulo, O; Reyes-Cordero, D; MoyaRoque, R. 2012. Estudio de la anatomía del xilema secundario de seis especies del género Citrus cultivadas en Costa Rica. Revista Forestal Mesoamericana Kurú (Costa Rica) 9(23):35-44.

Brito, JO; Barrichelo, LEG. 1977. Comportamento isolados da lignina e da celulose da madeira frente à carbonização. Instituto de Pesquisas e Estudos Florestais. Circular técnica no. $28.4 \mathrm{p}$

Brito, JO; Barrichelo, LEG. 1980. Correlações entre características físicas e químicas da madeira e a produção de carvão: 2 . Densidade da madeira $\mathrm{x}$ Densidade do carvão. Instituto de Pesquisas e Estudos Florestais IPEF no.20:101113

Chavesta, M. 2005. Maderas para pisos. Universidad Nacional Agraria la Molina. Lima, PE. 176p.

Chavesta, M. 2015. Atlas anatómico de maderas del Perú. Vol II.105p.

COPANT. 1972. Comisión Panamericana de Normas Técnicas. Maderas: Métodos para realizar el análisis estadístico de las propiedades de las maderas: Esquema $1^{\circ}$ de Norma. COPANT 30:1-012. 8p

Couto, A; Trugilho, PF; Napoli, A; Lima, JT; Silva, JR.; Protásio, T. 2015. Qualidade do carvão vegetal de Eucalyptus e Corymbia produzido em diferentes temperaturas finais de carbonização. Scientia Forestalis 43(108):817-831.

DGFFS. 2014. Dirección General Forestal y de Fauna Silvestre. Perú Forestal en Números año 2013. Lima, PE. 212p

Earl, D. 1975. Informe sobre el carbón vegetal. FAO. Roma. p.94

Gonçalves, T. 2010. Anatomia do lenho e do carvão de espécies arbóreas do Cerrado no es- 
tado de Sao Paulo, Brasil. Tesis Mag. Botucatu, BR. Universidade Estadual Paulista "Júlio de Mesquita Filho". 111 p.

Gonçalves, T; Nigoski, S; Oliveira, J; Marcati, C; Ballarin, A; Muñiz, G. 2015. A contribution to the identification of charcoal origin in Brasil II - Macroscopic characterization of Cerrado species. Anais da Academia Brasileira de Ciencias. 88(2):1045-1054.

Honorio, E; Aldana, D; Flores, G; Hidalgo, G; Mejía, E; Del Castillo, D; Humantupa, I; Baker, T; Degen, B; García, C. 2018. Fichas de identicación de las especies de Dipteryx de la Amazonía peruana. Instituto de Investigaciones de la Amazonía Peruana, Iquitos. 20 pp.

IAWA. 1989. International Association of Wood Anatomists. List of microscopic features for hardwood identification. Leiden, NL. V. 10 tomo 3, $333 \mathrm{p}$.

IBAMA. 1992. Instituto Brasileiro do Meio Ambiente e dos Recursos Naturais Renováveis. Norma de procedimentos em estudos de anatomia de madeira: I. Angiospermae, II. Gimnospermae. LPF-Serie Técnica Nº15. Brasilia. 5-15 p.

León W. 2014. Anatomía de maderas de 108 especies de Venezuela. VE. 264 p.

Muñiz, G; Nisgoski, S; Franca, R; Schardosin, F. 2012a. Anatomia comparativa da madeira e carvão de Cedrelinga catenaeformis Ducke e Enterolobium schomburgkii Benth. Para fins de identificacao. Scientia Forestalis, Piracicaba 40(94):291-297.

Muñiz, G; Franca, R; Fiorese, A; Nisgoski, S. 2013. Análisis de la estructura anatómica de la madera y del carbón de dos especies de Sapotaceae. Maderas. Ciencia y tecnología 15(3): 311-320.

Nigoski, S; Muñiz, G; França, R; Rodrigues, F; Batista, R. 2012. Anatomia do lenho carbonizado de Copaifera cf. langsdorfii Desf. e Dipteryx odorata (Aubl.) Wild. Ciencia da Madeira. $3(2): 66-79$.

Nigoski, S; Muñiz, G; Morrone, S; Schardosin, F; França, R. 2015. NIR and anatomy of wood and charcoal from Moraceae and Euphorbiaceae species. Ciencia da Madeira (Brazilian Journal of Wood Science) 6(3):183-190.

Pipa, E; Chavesta, M; Gonzales, H. 2009. Validación de una metodología de identificación del carbón vegetal del género Prosopis (algarrobo) a partir de la estructura anatómica. Anales científicos UNALM 70(2):132-136.

Souza, M; Silva, K; Perdigão, C; Silva, G; Melo, LE. 2015. Anatomia do lehno carbonizado de espécies madeireiras comercializadas na Amazonia. Fundação Casa da Cultura de Marabá no. 8.

Trugilho, P; Silva, D. 2001. Influencia de la temperatura final de carbonización en las características físicas y químicas del carbón vegetal de jatobá (Himenea courbaril L.). Scientia Agraria 2(1-2):45-53. 